\title{
Free-radical Carbo-functionalization of Olefins Using Sulfonyl Derivatives
}

\author{
Benjamin Ovadia, Frédéric Robert, and Yannick Landais*
}

\begin{abstract}
This review provides an overview of the recent progress made in the area of free-radical additions across the $\pi$-system of olefins, with a focus on the role of sulfonyl derivatives as one of the reaction partners in multicomponent processes. The mechanism and utility in organic synthesis of carbo-alkenylation, alkynylation, oximation and azidation of olefins are thus discussed and illustrated with some applications in total synthesis of natural products.
\end{abstract}

Keywords: Allylation · Azidation · Carbonylation $\cdot \beta$-Fragmentation $\cdot$ Oximation $\cdot$ Radical addition $\cdot$ Sulfones

\section{Introduction}

Olefins are used as readily available feedstock and important precursors for many transformations in organic synthesis. Their functionalization for instance through addition across the olefinic $\pi$-system has attracted considerable interest and constitutes a useful transformation in the armory of organic chemists nowadays. In parallel to transition-metal carbo-functionalization of olefins, freeradical addition to olefins has thus been recognized as a straightforward manner to access new synthons with increased functional complexity.[1] This review article will discuss the development of some free-radical additions of carbon fragments across the olefinic backbone, highlighting transformations using sulfonyl derivatives as final radical traps (Scheme 1). Further elaboration of the given intermediates to natural product synthesis will be described in a few cases and the mechanism will be detailed. Such transformations have been shown to rely strongly on polar effects, addition of the electrophilic $\mathrm{R}^{1}$ moiety occurring first onto the electron-rich olefin to generate a nucleophilic radical intermediate. The latter can then add efficiently to a variety of sulfonyl derivatives $\mathrm{ASO}_{2} \mathrm{Ph}$, where A represents either an halogen $(\mathrm{Cl})$, an azide, a cyanide or carbon fragments including olefins, alkynes or oximes.

\section{Seminal Work}

Fuchs, ${ }^{[2]}$ Kim and co-workers ${ }^{[3]}$ provided a strong basis for the development of radical carbo-functionalization of olefins using unsaturated sulfones as final radical traps. The former team discovered that simple triflone 1 reacted with an olefin through the incorporation of both a $\mathrm{CF}_{3}$ group and an alkynyl moiety across the olefinic $\pi$-system (Scheme 2). [2a] For instance, treatment of the $\alpha$-pinene 2 with AIBN led to the formation of alkyne $\mathbf{3}$ after addition of the $\mathrm{CF}_{3}$ radical onto the less hindered end of the olefin, followed by a fragmentation of the strained cyclobutane moiety and the trapping of the final tertiary radical center with triflone $\mathbf{1}$. Moderate to good yields were also obtained with a series of cyclic and acyclic olefins. The same year, Kim et al.[3] reported a study on the synthesis and reactivity of sulfonyl oximes such as 4 . Addition of C-centered radical onto 4 led to the corresponding oxime, which could then be converted into an aldehyde, this strategy thus constituting a straightforward one-carbon homologation and formally a formylation of an alkyl halide. Application of oxime 4 in a threecomponent process is highlighted below with the synthesis of highly functionalized cyclopentane 5 (Scheme 2).

Addition-fragmentation onto unsaturated sulfonyl systems may be generalized as depicted below with sulfonyl intermediate $\mathbf{I}$ (Scheme 3 ). The addition of a radical species to the $\alpha$-carbon of $\alpha, \beta$-unsaturated sulfones with subsequent departure of the sulfonyl group was established by Russell et al. ${ }^{[4]}$ The reaction occurs through the attack of the nucleophilic radical $\left(\mathrm{R}^{1}\right)$ onto the carbon center bearing the sulfonyl group and is followed by a $\beta$-fragmentation to release the final product and a sulfonyl radical II, which may evolve through two different pathways, depending on the nature of the $\mathrm{R}^{2}$ substituent. Arylsulfonyl radicals $\left(\mathrm{R}^{2}=\mathrm{Ar}\right)$ are known to be relatively stable and cannot sustain the radical chain alone. In this case, addition of $\mathrm{Bu}_{3} \mathrm{SnSnBu}_{3}$ to the sulfonyl radical is used to produce a $\mathrm{R}_{3} \mathrm{Sn}$ radical, which is able to propagate the radical chain. Alkylsulfonyl radicals are less stable and thus prone to $\alpha$-scission, ${ }^{[5]}$ with the formation of $\mathrm{SO}_{2}$ and an alkyl radical $\mathrm{R}^{2}$ which may react further. For instance, in Fuch's example above, the trifluoromethylsulfonyl group fragmented to afford $\mathrm{SO}_{2}$ and a $\mathrm{CF}_{3}$ radical $\left(\mathrm{R}^{2}\right)$, which then added to the olefin, thus sustaining the chain. Ethylsulfonyl radical was shown by Zard et al. ${ }^{[6]}$ to produce an ethyl radical which could then react with alkyl halides and xanthates $\left(\mathrm{R}^{1}-\mathrm{X}\right)$ to generate a new nucleophilic radical $\mathrm{R}^{1}$. Tin-free vinylation, allylation and oximation reactions were thus developed using this approach. All these processes rely on the match polarity between the vari- 
ous species present in the medium, therefore allowing multicomponent reactions to proceed smoothly without the formation of mixture of products.

We describe below recent efforts in our group and others to develop the chemistry of unsaturated sulfonyl derivatives in multicomponent functionalization of olefins. This review is not intended to be exhaustive but will focus on some significant progresses made in the domain.

\section{Addition onto $\mathbf{C}=\mathbf{C}$ Bonds (Allylation, Alkenylation, Alkynylation)}

\subsection{Carbo-allylation of Olefins}

1,4-Addition of nucleophilic species onto $\alpha, \beta$-unsaturated enones and enoates, followed by the trapping of the resulting enolate with an external electrophile constitutes a useful process in organic synthesis, which has seen many developments in the field of natural product synthesis. ${ }^{[7]} \mathrm{A}$ radical version was developed earlier by Mizuno, Otsuji and co-workers, ${ }^{[8]}$ using allylstannanes as final allylic trap. Similarly to its ionic counterpart, remarkable examples of diastereo- and enantioselective radical carbo-allylation have been reported using allylstannanes. ${ }^{[9]}$ Allylsulfones constitute excellent surrogates to allyltin reagents, which suffer from perceived toxicity and propensity to contaminate final products. Renaud et al. reported a useful tin-free tandem radical addition-allylation reaction of activated alkenes using organoboranes derivatives as radical precursors. ${ }^{[10]} \mathrm{A}$ nucleophilic alkyl radical, generated from the corresponding organoborane $\mathbf{6}$, was thus shown to react with an electron-poor olefin, e.g. maleimides or fumarates, affording the corresponding electrophilic radical adduct (Scheme 4). Allylation of this intermediate with allylsulfone $\mathbf{8}$, provided the allylating product $\mathbf{9}$, with a high level of diastereocontrol, after $\beta$-elimination of the phenylsulfonyl radical, sustaining the radical chain through reaction with the starting borane 6 .

Pioneering work by Zard using allylalkylsulfones such as $\mathbf{1 0}$ as an allylating agent is also worthy of note in this context (Scheme 5). ${ }^{[5 a]}$ Allybenzylsulfone $\mathbf{1 0}$ was thus shown to add across the double bond of olefin 11 with loss of $\mathrm{SO}_{2} . \alpha$-Scission of the thiophenemethylsulfonyl radical furnished a nucleophilic radical which added onto the electron-deficient olefin 11, leaving an electrophilic radical which was finally allylated with $\mathbf{1 0}$ to afford $\mathbf{1 2}$ in moderate yield.

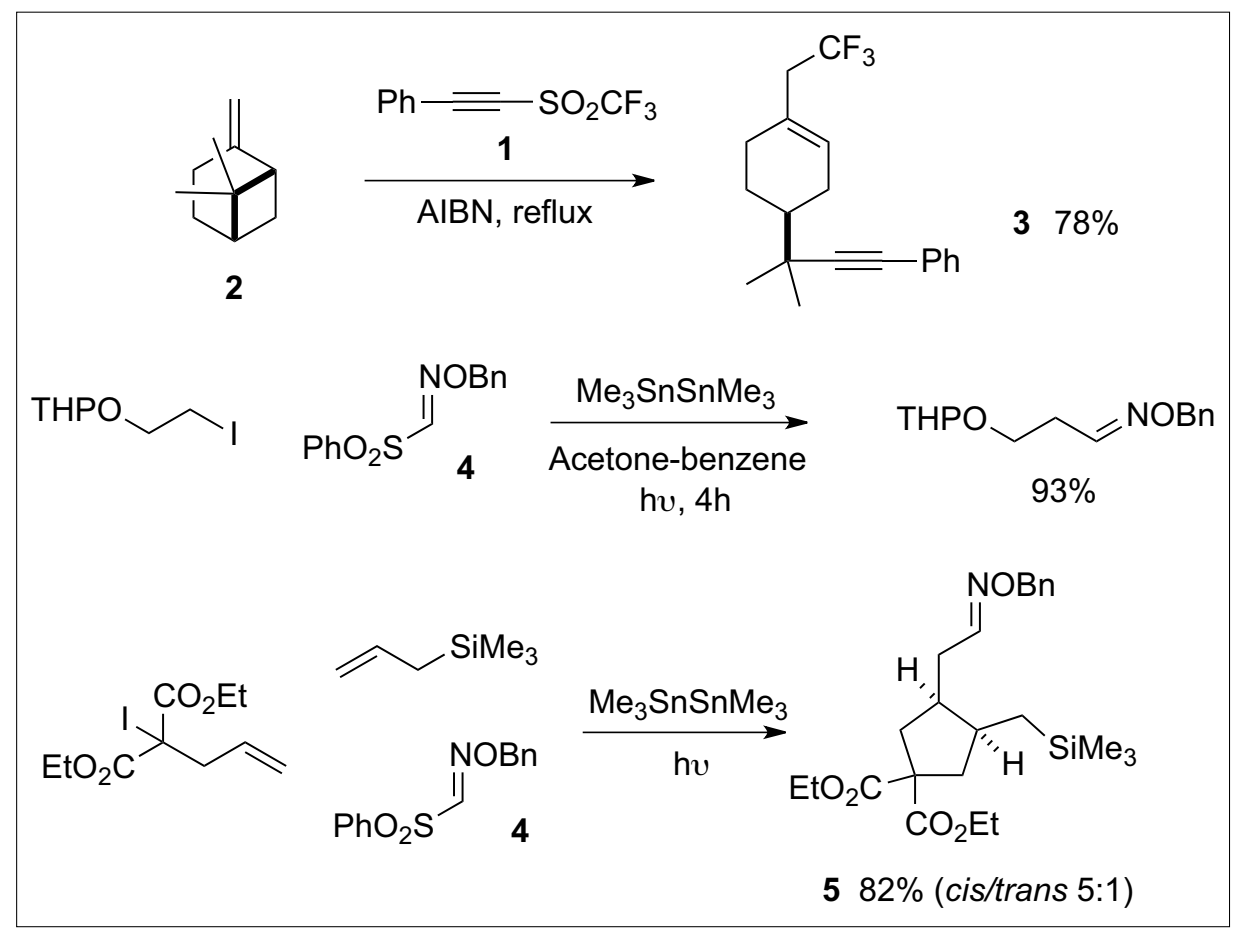

Scheme 2. Pioneering studies from Fuchs and Kim and co-workers.

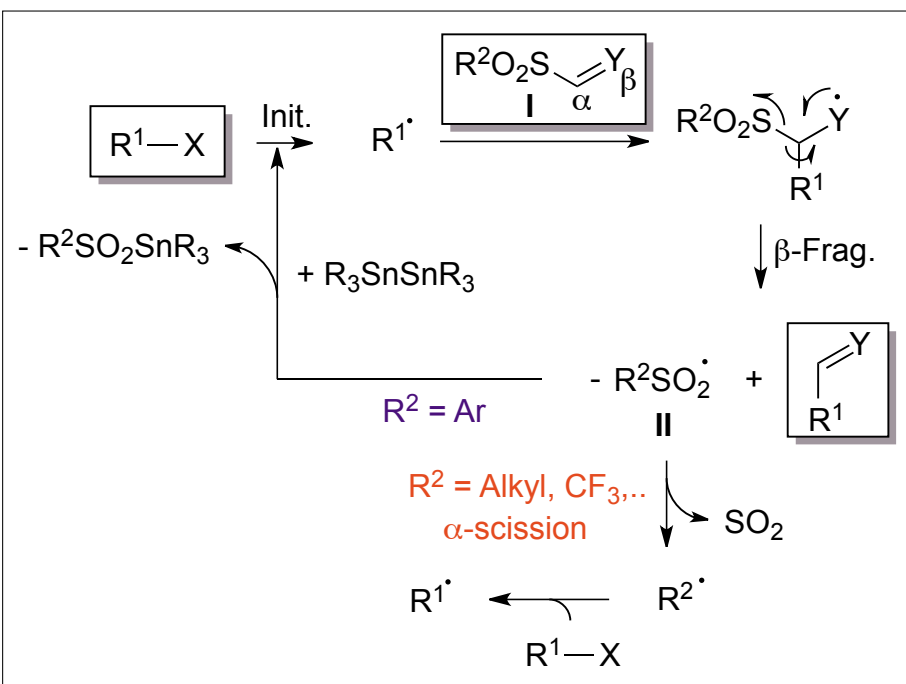

Scheme 3. Additionfragmentation processes using sulfonyl derivatives.

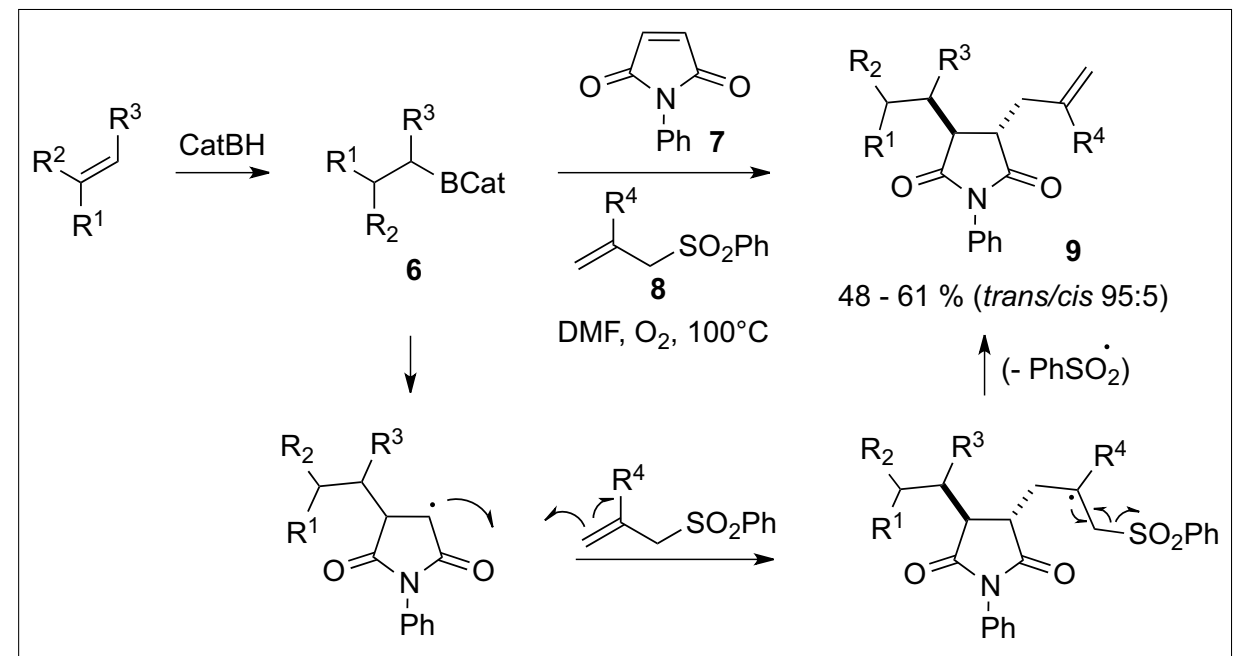

Scheme 4. Carbo-allylation of activated olefins. 


\subsection{Carbo-alkenylation and Alkynyl- ation of Olefins}

Pioneering studies by Fuchs et al. have shown that alkenylation and alkynylation of ethers or alkanes, using vinyl- and alkynyltriflone derivatives 13 and 14, were efficient processes (Scheme 6). ${ }^{[2]}$ The reaction proceeds through radical $\mathrm{C}-\mathrm{H}$ abstraction by the electrophilic trifluoromethyl radical,[2a,11] followed by the addition of the resulting nucleophilic alkyl radical onto the alkenyl- or alkynyltriflone with $\beta$-elimination of the trifluoromethanesulfonyl radical. The process relies on the formation of the strong $\mathrm{CF}_{3}-\mathrm{H}$ bond. Trifluoromethyl-mediated carbonylative alkynylation of alkane was later reported by Ryu following the same concept.[11]

As discussed above, triflones $\mathbf{1 3}$ and 14 were also used to carry out trifluoromethyl-alkynylation of olefins. However, this method was limited to the incorporation of a $\mathrm{CF}_{3}$ group as the electrophilic radical precursor. We recently developed a new free-radical three-component carboalkenylation and alkynylation of olefins, which resulted in the addition of two functional groups across the $\pi$-system of unactivated olefins. ${ }^{[12]}$ This process relies on the addition of an electrophilic radical species III, generated from the corresponding xanthates or alkyl halides, to the less hindered end of an electron-rich olefin (Scheme 7). The resultant nucleophilic radical IV can then be trapped by the electrophilic vinylor alkynylsulfone acceptor. The phenylsulfonyl radical released during the addition$\beta$-elimination sequence, which does not fragment under the reaction conditions (e.g. $65^{\circ} \mathrm{C}$ ), serves as a radical chain propagator in the presence of a stoichiometric amount of hexabutylditin.

The carbo-alkenylation products were obtained with excellent yields using the reactive ethylene bisphenylsulfone 15. ${ }^{[13]}$ Slightly lower yields were observed with less electrophilic sulfone 16. As expected, electron-rich olefins including allylsilanes or vinyl ethers were more reactive, emphasizing the crucial role of polar effects in this process. ${ }^{[14]}$ Mono- and disubstituted alkenes also led to the desired adducts with reasonable to good yields. Quaternary centers could thus be generated using $1,1^{\prime}$-disubstituted olefins. The nature of the radical precursor (xanthate or alkyl halide) could also be varied, providing an access to different ketones, nitriles, amides or malonates in good yields (Scheme 8).

Recent studies also provided information relating to the role of the phenylsulfonyl radical released during the addition onto the vinylsulfone. ${ }^{[15]} \mathrm{PhSO}_{2}$ is a very electrophilic radical, which also adds efficiently onto olefins (generally used in excess in the multicomponent process). Fortunately, this addition is reversible lead-

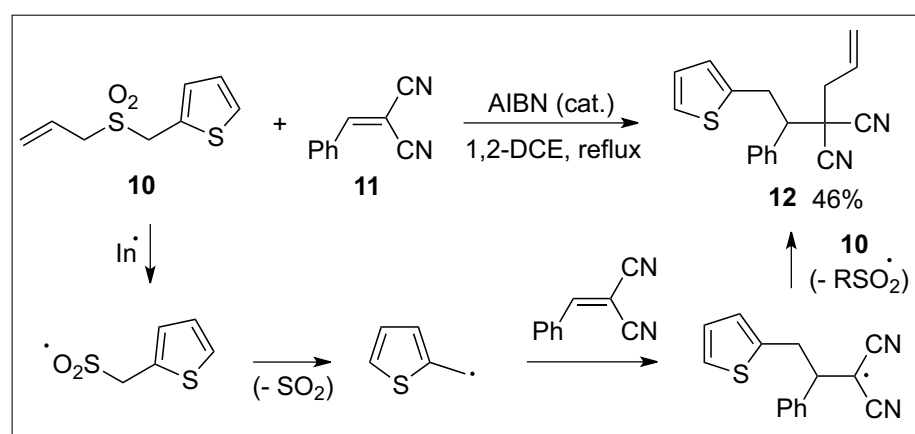

Scheme 5. Addition of an allylalkylsulfone across an olefin double bond.
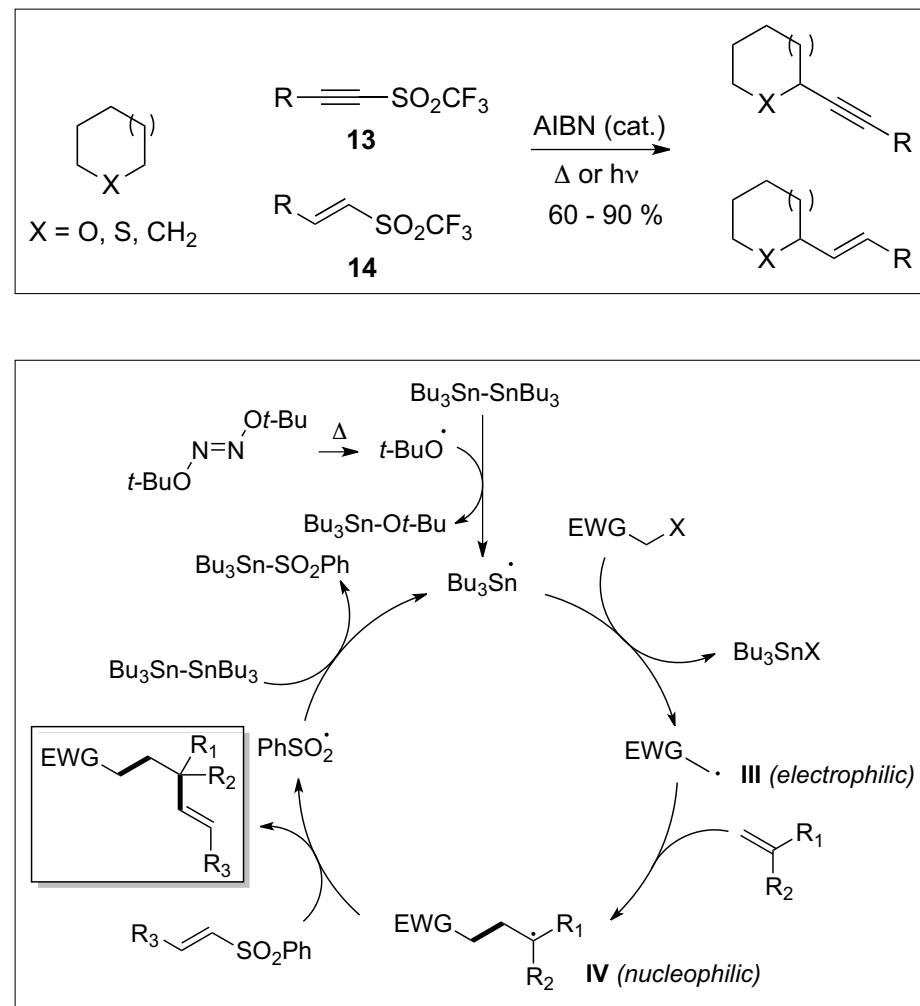

Scheme 7.

Mechanism of the carbo-alkenylation of olefins.

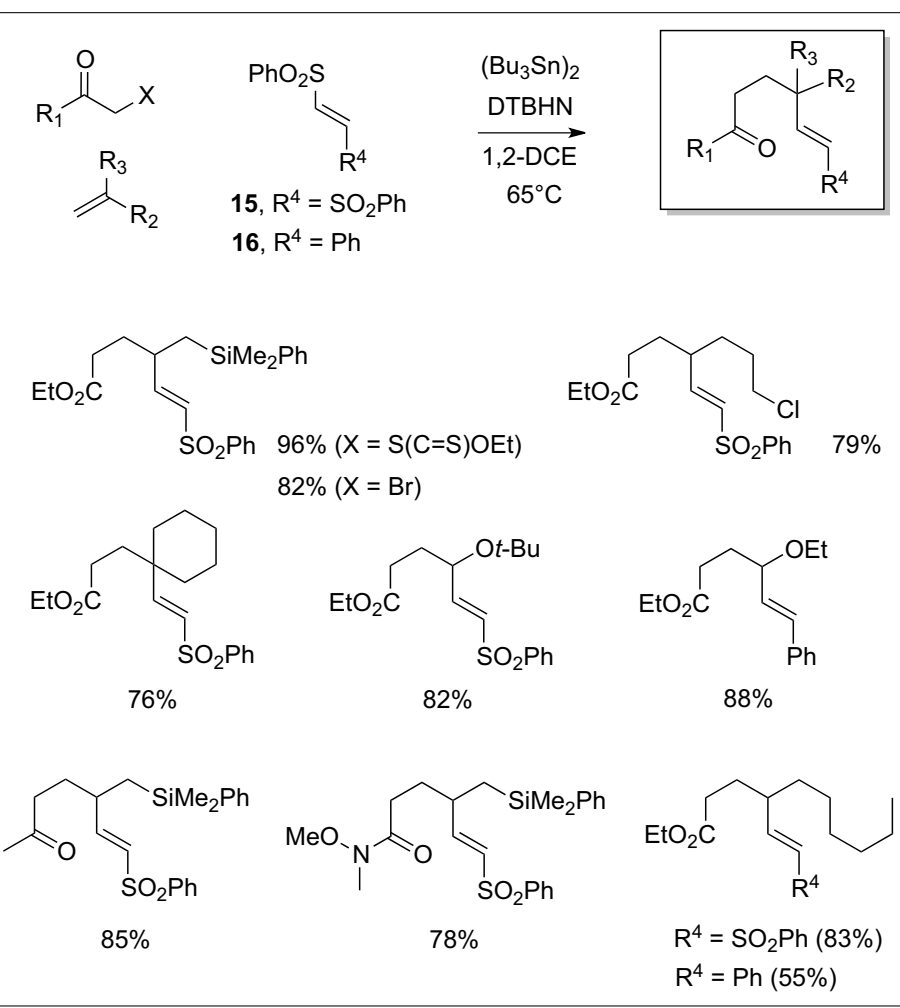

Scheme 8. Carboalkenylation of olefins. 


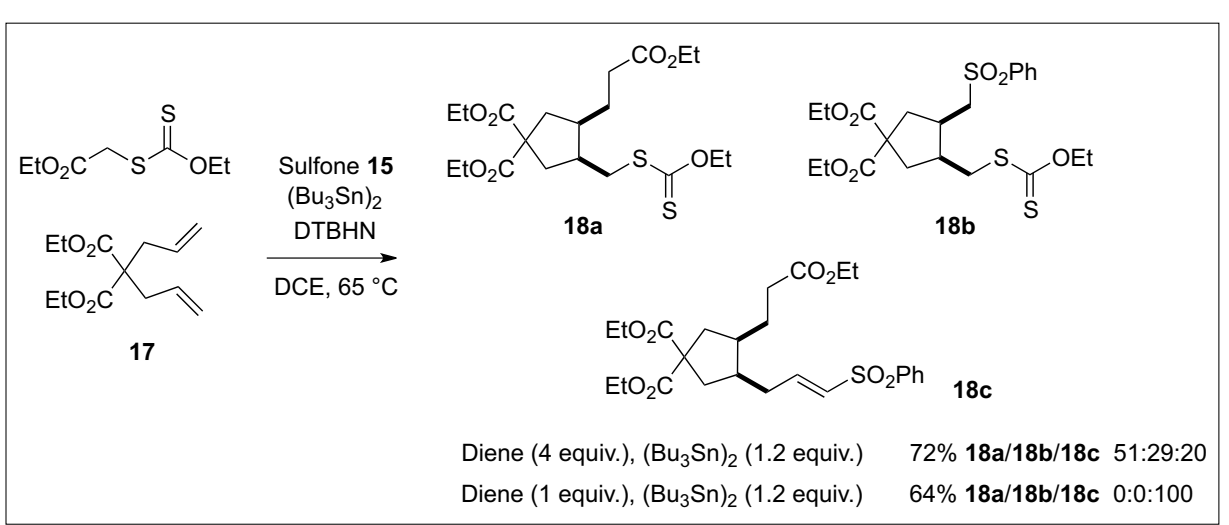

yields dropped significantly. Contrary to the acyclic systems, and in contrast with the observations made during addition of similar radicals to enamines, no stereocontrol was observed with acyclic ene-carbamates. ${ }^{[17]}$

Enamides react similarly to provide functionalized lactams with high stereocontrol. These compounds may then be functionalized further, as illustrated with the pyrrolidine-mediated cyclization of ketone 19 into aza-decalone 20, a potent building block in the synthesis of alka-

Scheme 9. Carbo-alkenylation of dienes.

ing back to the olefin. ${ }^{[16]}$ The rate of the $\beta$-fragmentation is thus crucial for the success of the carbo-alkenylation. ${ }^{[16 c]}$ A slow $\beta$-fragmentation leads to an accumulation of a sulfonyl addition product, as shown by the carbo-alkenylation of dienyl model 17 (Scheme 9). Using an excess of diene provided the addition product $18 \mathbf{a}$, resulting from an addition of the xanthate onto $\mathbf{1 7}$, followed by a xanthate group transfer. In turn, the formation of $\mathbf{1 8 b}$ resulted from the addition of the $\mathrm{PhSO}_{2}$ radical onto 17, which was followed by a fast 5-exotrig reaction and transfer of xanthate. The fast cyclization thus outcompeted the $\beta$-fragmentation of the $\mathrm{PhSO}_{2}$ group. Starting from a stoichiometric amount of the three partners finally led to the unique formation of the desired carbo-alkenylation product 18c.

We recently extended the scope of application of the three-component carbo-alkenylation of olefins to cyclic and acyclic enamides and ene-carbamates, offering access to a wide range of highly functionalized amides and carbamates. ${ }^{[17]}$ These precursors are versatile intermediates for the synthesis of heterocycles, amino acids or other building blocks for the construction of complex natural products. In contrast to the reaction under ionic conditions, where enamides and ene-carbamates exhibit nucleophilic then electrophilic reactivity on the $C(2)$ and $C(1)$ carbons respectively, ${ }^{[18]}$ as in Scheme 10a, radical conditions offer a complementary set of reactivity. The $\mathrm{C}(2)$ center reacts efficiently with an electrophilic radical precursor as already mentioned before, leading to an electron-rich radical intermediate, which can then be trapped by the third electrophilic partner (Scheme 10b).

The unusual ene-carbamate reactivity was illustrated with the reaction of various xanthates with acyclic and cyclic ene-carbamates and vinyldisulfone $\mathbf{1 5}$ as the electrophilic partner (Scheme 11). Carbo-alkenylation products were generally obtained in high yields and diastereocontrol in the cyclic series. Introduction of a quaternary center was possible, albeit in these cases (a) Ionic reactivity

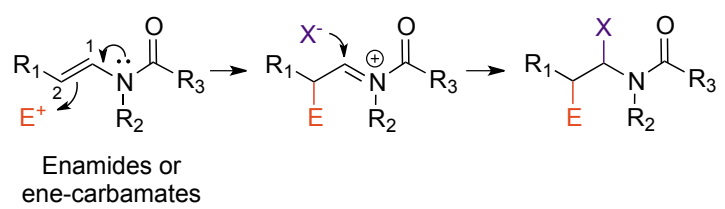

(b) Radical reactivity

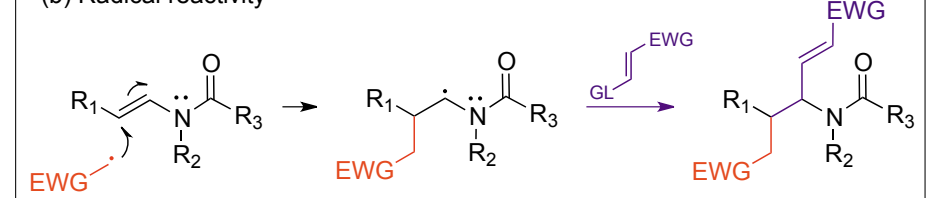
EWG

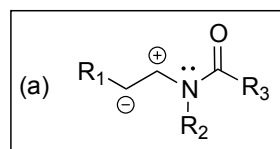

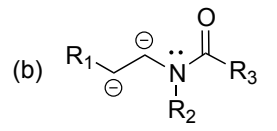

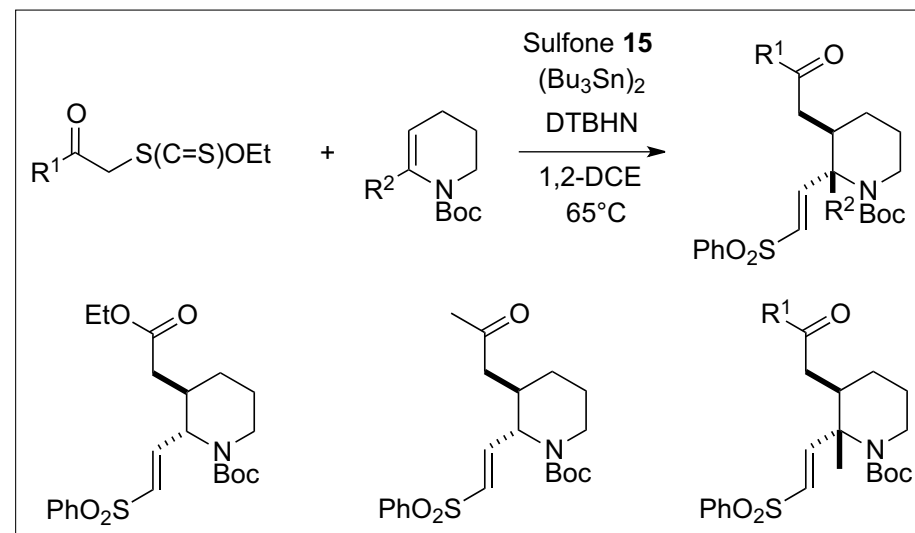

$85 \%$ (d.r. $>98:<2$ )

$74 \%$ (d.r. $>98:<2$ )
Scheme 10. Ionic and radical reactivities of enamides and enecarbamates.

Scheme 11. Carboalkenylation of enecarbamates.

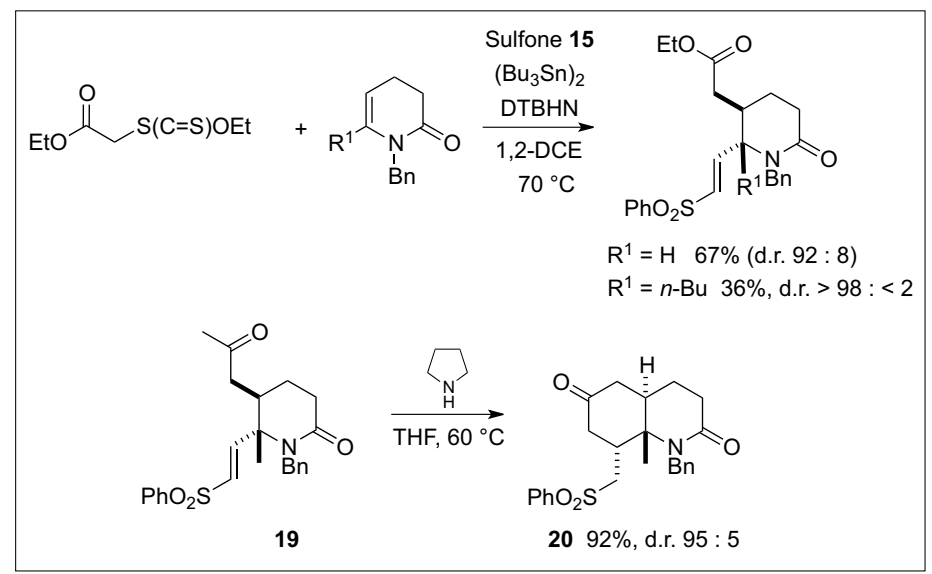

Scheme 12. Carboalkenylation of enamides. 


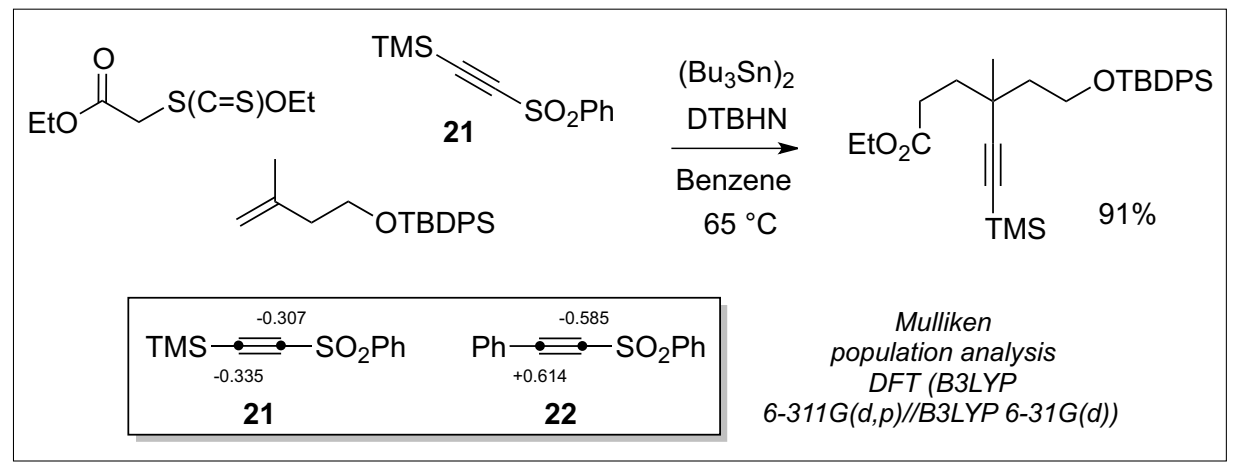

Scheme 13. Carbo-alkynylation of olefins.

loids of the lycopodium family, ${ }^{[19]}$ which was obtained as a single diastereoisomer (Scheme 12).

Using similar conditions, our laboratory devised a carbo-alkynylation reaction starting from alkynylsulfone $\mathbf{2 1}$ as an electrophilic trap. ${ }^{[12]}$ Carbo-alkynylation products were obtained in moderate to excellent yields using different activated and unactivated olefins (Scheme 13). Better results were generally observed with alkynylsulfone $\mathbf{2 1}$ bearing a trimethylsilyl substituent, as compared with the phenyl analogue 22. This was attributed to a lower polarization in silylalkyne $\mathbf{2 1}$ due to a strong $\pi$-d interaction between silicon and the carbon center. ${ }^{[20]}$

\section{Carbo-oximation of Olefins}

As discussed above, sulfonyloximes such as $\mathbf{4}$ are good radical traps, and constitute useful tools to incorporate a masked carbonyl group in a carbon framework. [3] Ryu and Kim have developed remarkable carbonylative processes involving sulfonyloximes 4 and 23. ${ }^{[21]}$ Scheme 14 illustrates such a carbonylative cyclization, which is terminated by the trapping of the final nucleophilic acyl radical by oxime 23 . Interestingly, the $\mathrm{PhSO}_{2}$ radical released in the final step reacts with the electron-rich allyltin reagent, generating the $\mathrm{Bu}_{3} \mathrm{Sn}$ radical which can then abstract the iodine from the starting iodo-olefin and thus propagate the chain. A related tin-free process was developed recently in this laboratory using allylsilanes and allylthiosilanes as allyltin surrogates. ${ }^{[22]}$

The oxime introduced upon the reaction with a nucleophilic radical species may then be engaged in further transformations. We devised recently a sequential four-component reaction, in which the oxime was shown to react with an alkyl radical or an allylzinc reagent, these additions then triggering a lactamization and a one-pot formation of 5,6-disubstituted piperidinones in good yield and high diastereoselectivities (Scheme 15).[23] This method represents a straightforward strat- egy to access, in a one-pot operation, the piperidine skeleton, ubiquitous in complex natural alkaloids.

Generalization of this three-component carbo-oximation reaction of olefins constitutes a formal carbo-formylation process using sulfonyloxime $\mathbf{4}$ as a non-toxic $\mathrm{CO}$ surrogate. It is also worth adding that addition of nucleophilic radical species to 4 generally occurs with high rate constant (e.g. $10^{6} \mathrm{M}^{-1} \mathrm{~s}^{-1}$ for a primary alkyl radical), ${ }^{[25]}$ avoiding the use of high pressure of $\mathrm{CO}$ and specific autoclave equipment. The scope of application of the three-component carbo-oximation of electron-rich olefins is rather broad, reaction conditions being compatible with many functional groups (esters, ketones, amides, thioethers, silyl ethers, nitriles, halogens, ....). The reaction also worked well with gem-disub-
Scheme 15. Three-component carbo-oximation of olefins. stituted olefins, leading to the formation of quaternary centers. New oximes such as $\mathbf{2 4}$ were also devised, which were found to be slightly more reactive than $\mathbf{4}$ and could be hydrolyzed under milder conditions (TFA, $\left.\mathrm{CH}_{2} \mathrm{Cl}_{2}, \mathrm{RT}\right)$. ${ }^{[24]}$ The subsequent hydrolysis of the final oximes revealed the aldehyde function which was then engaged in various nucleophilic cascades such as Sakurai allylation/lactonization or domino Mukaiyama aldol condensation/lactonization, allowing the formation of highly functionalized lactones. A Pictet-Spengler/lactamization sequence also opened an access to the tetracyclic core $\mathbf{2 5}$ of eburnan alkaloids (Scheme 16). ${ }^{24]}$

The $\mathrm{PhSO}_{2}$ radical released during the addition- $\beta$-fragmentation sequence above does not fragment under the reaction conditions $\left(70-80^{\circ} \mathrm{C}\right)$ to furnish a phenyl radical which could sustain the radical chain. A stoichiometric amount of hexabutylditin is thus required, generating large amounts of tin residues which contaminate the final products. Based on precedent from Zard[5a] and Renaud's groups, ${ }^{[26]}$ we devised new bifunctional reagents $\mathbf{2 6 a}-\mathbf{e}$, incorporating both the oxime function and the radical precursor moiety, thus avoiding the use of tin additives. ${ }^{[27]}$ These reagents act both as traps for nucleophilic radicals as well as sources of electrophilic radicals via the $\alpha$-scission of the alkylsulfonyl radical intermediates. This reversible $\alpha$-scission, ${ }^{[28]}$ relatively easy with alkylsulfonyl species,

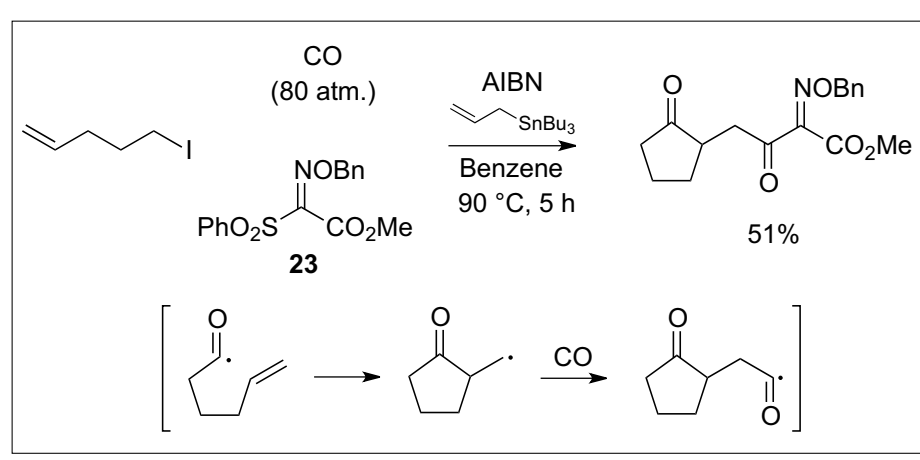

Scheme 14. Carbonylation-oximation of alkyl halides.
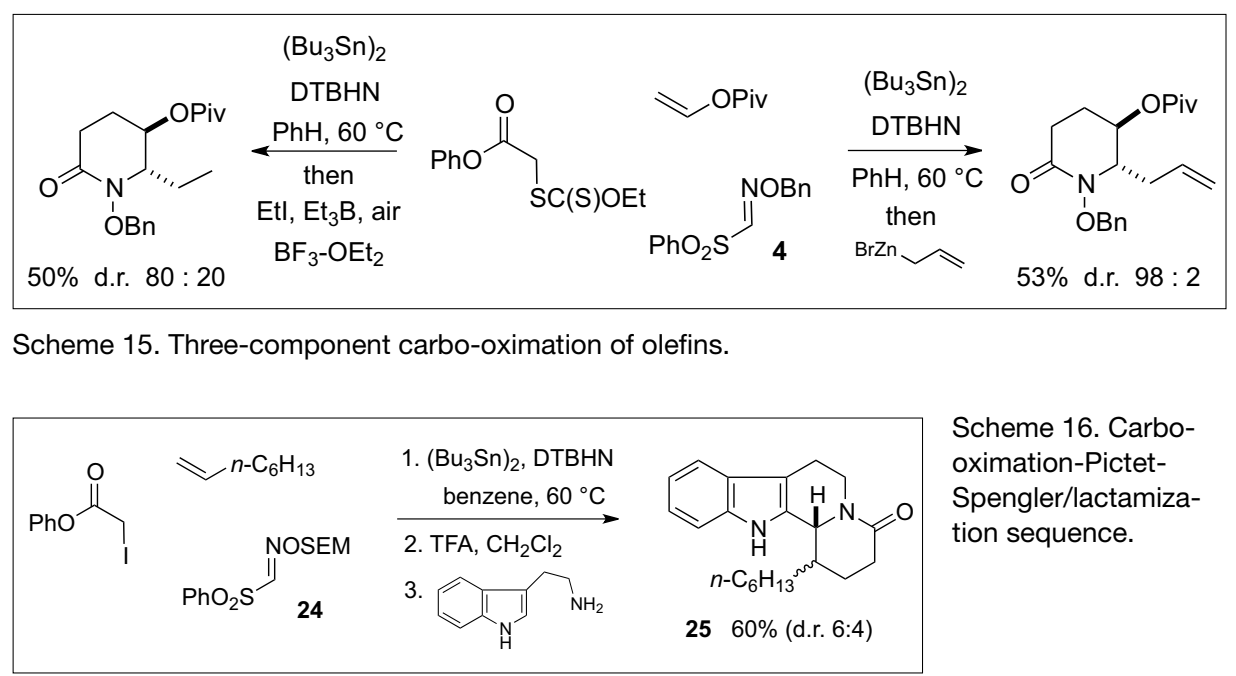

Scheme 16. Carbooximation-PictetSpengler/lactamization sequence. 
leads to an alkyl radical which then adds onto the olefin, generating a nucleophilic radical which finally reacts with $\mathbf{2 6 a}-\mathbf{e}$ to propagate the chain (Scheme 17). The addition of a carbon-centered electrophilic radical and an oxime across the double bond of an electron-rich olefin proceeded with moderate to good yields, oximes 26a-e exhibiting modest reactivity as compared to oximes $\mathbf{4}$ or $\mathbf{2 4}$, for steric reasons. Although the process could be carried out under tin-free conditions, a catalytic amount of hexabutylditin was, however, shown to increase the yield. The initiation step using DTBHN followed an unusual mechanism supported both by experiment and DFT calculations. The benzylic hydrogen of the oxime ether is believed to be abstracted first by a $t$-BuO radical, the $\beta$-fragmentation of the resulting benzylic radical then, releasing benzaldehyde, cyanhydric acid and $\mathrm{SO}_{2}$ to generate the first electrophilic radical I (Scheme 17). Following this pathway, tin is not required. The role of the catalytic amount of tin is not yet clear, but an attack of the nucleophilic tin radical onto the sulfonyl oxime 26, followed by a $\beta$-fragmentation step appears to be a plausible competitive pathway to generate the electrophilic radical $\mathbf{I}$.

\section{Carbo-azidation of Olefins}

In 2001, Renaud and co-workers first reported the use of sulfonylazides such as $\mathbf{2 7}$ as radical traps for the formation of $\mathrm{C}-\mathrm{N}$ bonds, as illustrated with the cyclization-azidation sequence below (Scheme 18).[29] The electrophilic $\alpha$-enoyl radical issued from the abstraction of the iodide in $\mathbf{2 6}$ by a tin radical underwent a 5-exo-trig cyclization to generate a new nucleophilic radical which was then trapped by the electrophilic phenylsulfonyl azide to form the bicyclic azide $\mathbf{2 8}$ in good yield as a mixture of two diastereomers.

The electrophilic character of $\mathbf{2 7}$ is key to the success of the azidation step. Azide 27 reacts very efficiently with nucleophilic radicals and much slower with ambiphilic or electrophilic radicals. This strong polar effect thus led the authors to extend the intramolecular azidation process to a threecomponent carbo-azidation reaction, ${ }^{[30]}$ the mechanism of which being related to that of carbo-alkenylation and alkynylation of olefins (Scheme 7). The one-pot addition of an electrophilic carbon centered radical onto the less hindered end of an unactivated olefin, followed by the trapping of the resulting nucleophilic radical with 27 was shown to be very efficient, allowing the formation of azides $\mathbf{2 9}$ with moderate to good yields (Scheme 19).

The utility of the carbo-azidation reaction was illustrated with the straightforward

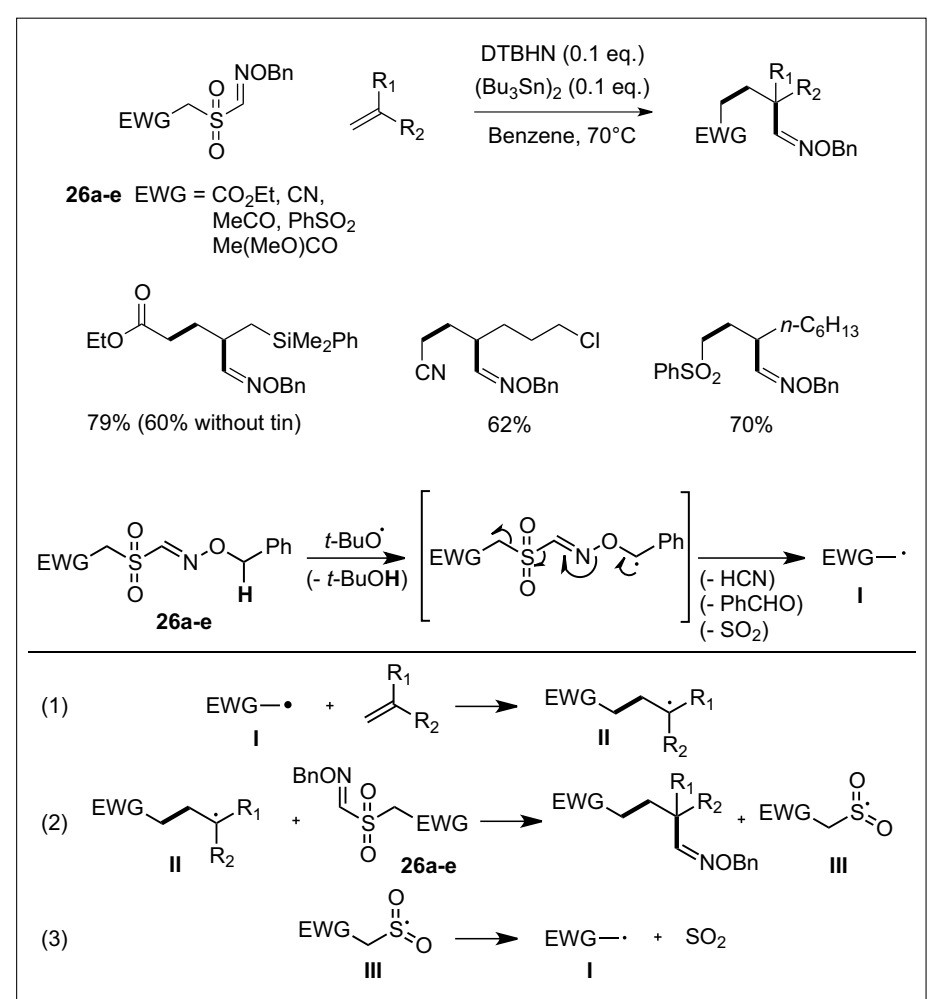

Scheme 17. Twocomponent carbooximation. Initiation process.

and efficient synthesis of pyrrolizidine and indolizidine alkaloid skeleton. The synthesis of lepadiformine was thus carried out

based on the carbo-azidation of olefin 30, which afforded the key intermediate $\mathbf{3 1}$ in good yield, albeit with poor stereocontrol
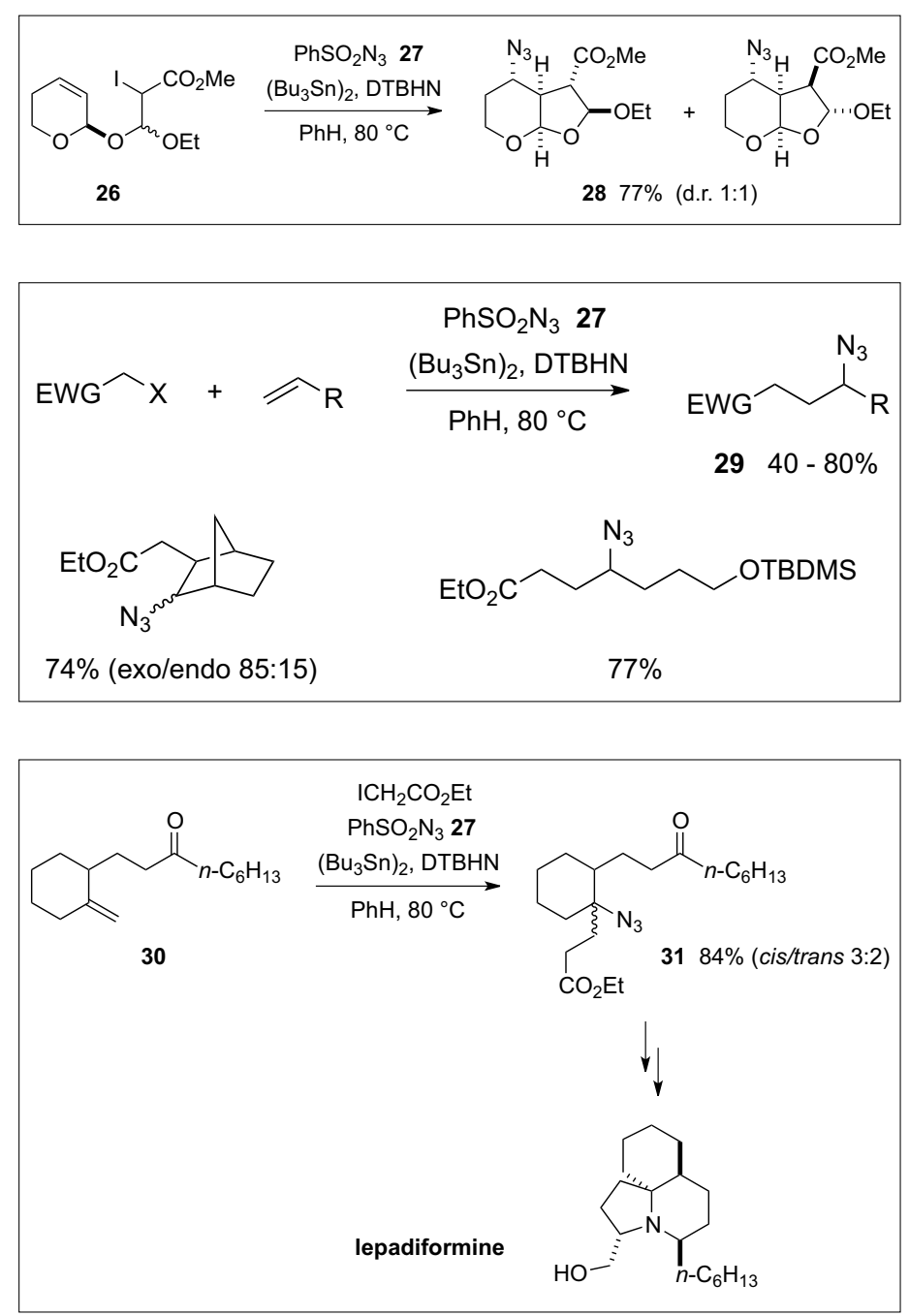

Scheme 18. Intramolecular carboazidation of olefins.

Scheme 19. Intermolecular carboazidation of olefins.

Scheme 20. Carboazidation in lepadiformine synthesis. 
(Scheme 20). ${ }^{[31]}$ The stereocontrol issue in carbo-azidation of substituted methylenecyclohexane (e.g. 30) was studied in depth, the linear sulfonyl azide generally favoring an axial approach anti to the allylic substituent (except with bulky substituents) to minimize torsional strain and 1,3-diaxial interactions. ${ }^{[32]}$

In the frame of a collaborative program between this laboratory and the team of Prof. P. Renaud in Bern, we studied the stereocontrol during the carbo-azidation of acyclic chiral allylsilanes (Scheme 21). Addition of xanthates onto electron-rich allylsilanes, possessing one or two stereogenic centers gave rise to a $\beta$-silyl radical, which was finally trapped by the sulfonyl azide 27. This led to the formation of a range of $\beta$-azidosilanes with a high level of diastereocontrol (up to 95:5 in favor of the syn isomer), depending on the nature of the silicon group and that of the $\mathrm{R}^{1}$ substituent (Scheme 21). [33]

The treatment of the major diastereoisomer with a fluoride source led exclusively to the (Z)-alkene. Assuming an antistereospecific Peterson-like elimination, we were able to correlate the $(Z)$-configuration of the alkene with the formation of the syn $\beta$-azidosilanes as major isomers. ${ }^{[34]}$ This result, supported by DFT calculations and X-ray studies, was rationalized invoking a Felkin-Ahn type model with a pyramidalized transition state and a quasistaggered conformation to avoid gauche interactions between the most hindered groups $\mathrm{SiR}_{3}$ and $\left(\mathrm{CH}_{2}\right)_{2} \mathrm{CO}_{2} \mathrm{Et}$ (Scheme 21). ${ }^{[35]}$ The sulfonyl azide 27 is believed to approach anti relative to the bulky silicon group from the side of the smallest $\mathrm{H}$ substituent. The formation of the minor diastereomer would result from an approach of 27 anti relative to the silicon group, but close to the bulky R2 substituent. It is also worth of note that in such a transition state, the developing positive charge $\beta$ to silicon is best stabilized by the quasi coplanar electron-rich $\mathrm{C}$-Si bond (silicon $\beta$-effect). This stereocontrol in carbo-azidation of chiral acyclic allylsilanes was a key feature in the first enantiocontrolled synthesis of hyacinthacine $A_{1}$, a polyhydroxylated pyrrolizidine from Muscari Armeniacum (Scheme 22). ${ }^{[36]}$

\section{Miscellaneous Processes Involving Sulfonyl Derivatives}

Multicomponent processes involving a carbonylation step have been reported by Ryu and co-workers using sulfonyl derivatives as final radical traps. ${ }^{[37]}$ Formation of thioesters was thus developed starting from both an allylsulfone $\mathbf{3 4}$ and phenylsulfonylthioether $\mathbf{3 5}$ in the presence of carbon monoxide under high pressure

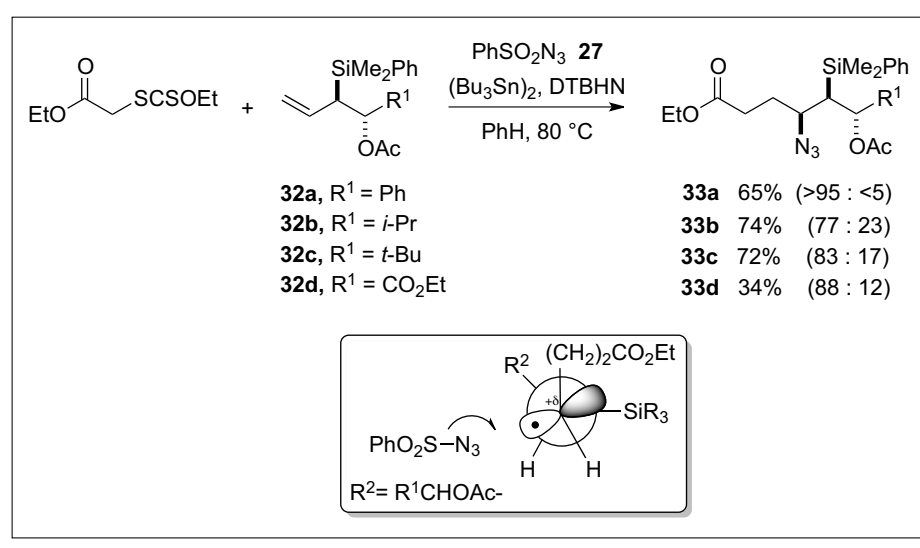

Scheme 21. Diastereocontrolled carboazidation of allylsilanes.

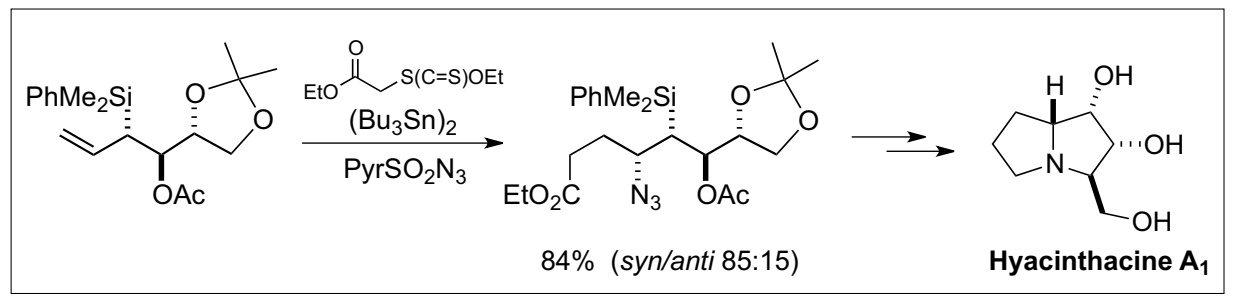

Scheme 22. Carbo-azidation in hyacinthacine $A_{1}$ synthesis.

(Scheme 23). The nucleophilic radical resulting from the reaction of $\mathbf{3 4}$ with initiator V-40 was shown to react with carbon monoxide, producing a nucleophilic acyl radical, which was finally trapped by the sulfonyl thioether 35, affording the desired thioester in moderate to excellent yields, along with the $\mathrm{PhSO}_{2}$ radical, which adds to $\mathbf{3 4}$, thus propagating the radical chain. The success of the process depends of the amount of alkyl sulfide formed upon trapping of the nucleophilic alkyl radical by the electro-deficient acceptor. In practice, high pressure of $\mathrm{CO}$ is required to decrease the amount of alkyl sulfide resulting from the direct reaction of the nucleophilic alkyl radical with $\mathbf{3 5}$. Secondary and tertiary alkyl radicals gave a mixture of the desired product with a significant amount of the alkyl sulfide, due to the competitive fast decarbonylation occurring with secondary and tertiary acyl radicals. Higher pressure of CO (130 atm) was shown to decrease the formation of the side product in these cases. Based on the same process, Kim, Ryu and co-workers extended the thiocarbonylation to the tin-free cyanocarbonylation using sulfonylcyanide $\mathbf{3 6}$ as a radical trap (Scheme 23).[38]

The above process was extended to a remarkable four-component carbonylation process involving an allylsilane and affording a convenient access to functionalized cyclopentanes bearing a thioester functional group, with the formation of four new bonds (3 C-C and $1 \mathrm{C}-\mathrm{S}$ ) (Scheme 24). ${ }^{[37]}$ The electrophilic malonyl radical, generated from the alkyl allylsulfone 37, failed to undergo carbonylation and preferentially reacted with the electron-rich allylsilane. Subsequent 5-exo-trig radical cyclization, then carbonylation produced an acyl radical which was trapped by sulfonylthiother 35, affording the desired thioester in high yield. As above, the electrophilic $\mathrm{PhSO}_{2}$ radical was shown to react efficiently with allylsulfone 37, thus sustaining the radical chain.

Finally, Renaud et al. have developed recently a 'carbo-chlorination' of olefin using a chlorosulfone 38 (Scheme 25). [39] The latter reacts similarly to sulfonyloximes 26 (Scheme 17). Initiation with DLP produced an alkylsulfonyl radical, which after $\alpha$-scission added to the olefin, generating a nucleophilic radical, reacting in a final step with $\mathbf{3 8}$ to form the $\mathrm{C}-\mathrm{Cl}$ bond. The reaction was found to be general, leading to good yields of the corresponding chlorides. This atom transfer radical addition (ATRA) is particularly attractive and competes efficiently with transition-metal mediated ATRA processes, ${ }^{[40]}$ as sulfonyl chlorides are excellent radical chlorinating agents, reacting with high rate con-

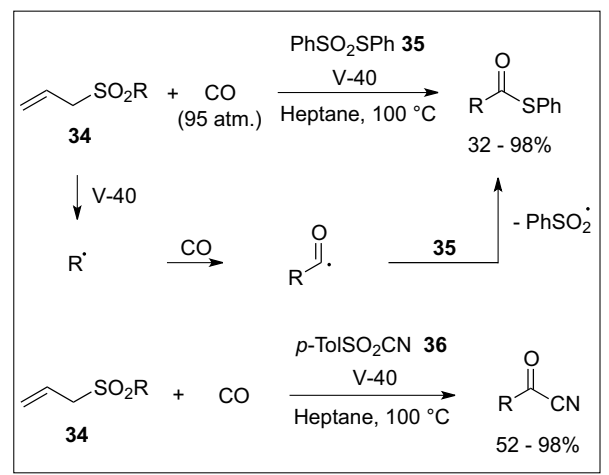

Scheme 23. Phenylthioester and acylcyanide synthesis through carbonylation and addition of sulfonyl derivatives. 

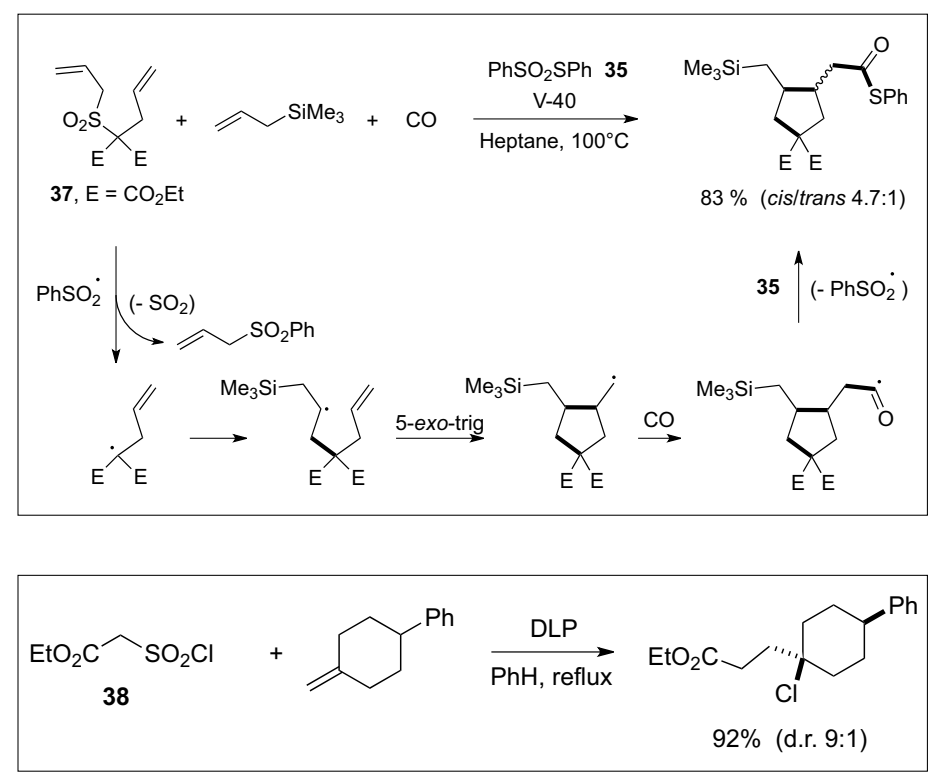

stants with C-centered radical species (for instance, primary radical reacts with such chlorinating agent with $\mathrm{k}=1.310^{6} \mathrm{M}^{-1} \mathrm{~s}^{-1}$ at $\left.25^{\circ} \mathrm{C}\right)$. ${ }^{[41]}$

\section{Conclusion}

In summary, sulfonyl reagents allow the transfer of a wide range of substituents, from halogens $(\mathrm{Cl}, \mathrm{Br})$ to chalcogen $(\mathrm{SPh}$, $\mathrm{SePh}$ ) to unsaturated functions including azides, alkenes, alkynes and oximes. The electrophilic nature of the sulfonyl radicals also allow their reaction with electron-rich olefins, as illustrated for instance in azidosulfonation of olefins and dienes. ${ }^{[42]}$ The sulfonyl radicals may also react with alkylboranes, liberating an alkyl radical which may then be functionalized, this strategy notably expanding the value of the hydroboration reaction. Tin-free processes have been developed, relying on the ability of alkylsulfonyl intermediates to $\alpha$-fragment, liberating $\mathrm{SO}_{2}$ and a new C-centered radical. Recent studies based on the oxidation of the Langlois reagent $\mathrm{CF}_{3} \mathrm{SO}_{2} \mathrm{Na}$ into the corresponding trifluoromethanesulfonyl radical and the subsequent formation of the $\mathrm{CF}_{3}$ radical have not been discussed here but demonstrate that such reagents still hold much promise for further developments. ${ }^{[43]}$ More generally, oxidation of reagents of the type $\mathrm{RSO}_{2}$-Metal $(\mathrm{M}=$ $\mathrm{Zn}, . .$. .) as a source of alkyl radicals has recently generated a high interest. ${ }^{[44]}$

\section{Acknowledgements}

We thank the CNRS, the University of Bordeaux and the Agence Nationale de la Recherche for financial support. We are also grateful to Prof. P. Renaud (University of Bern), Prof. I. Ryu and Prof. T. Fukuyama (University of Osaka Prefecture) and Prof. M. Heinrich (University of Erlangen) for fruitful discussions during collaborative work in
Scheme 25. Carbochlorination of olefins.

Scheme 24. Fourcomponent carbonylation process and addition of $\mathrm{PhSO}_{2} \mathrm{SPh}$.

.

he frame of the following research programs: PHC-Germaine de Staël (CH), PHC-Sakura (Japan) and PHC-Procope (D).

Received: May 26, 2015

[1] H. Subramanian, Y. Landais, M. P. Sibi in 'Comprehensive Organic Synthesis', Eds. G. A. Molander, P. Knochel, $2^{\text {nd }}$ ed., Vol. 4, Oxford: Elsevier, 2014, pp. 699-741.

[2] a) J. Gong, P. L. Fuchs, J. Am. Chem. Soc. 1996, 118, 4486; b) J. Xiang, P. L. Fuchs, $J$. Am. Chem. Soc. 1996, 118, 11986; c) J. Xiang, W. Jiang, J. Gong, P. L. Fuchs, J. Am. Chem. Soc. 1997, 119, 4123; d) J. Xiang, J. Evarts, A Rivkin, D. P. Curran, P. L. Fuchs, Tetrahedron Lett. 1998, 39, 4163; e) J. Xiang, P. L. Fuchs, Tetrahedron Lett. 1996, 37, 5269; f) J. Xiang, W. Jiang, P. L. Fuchs, Tetrahedron Lett. 1997, 38,$6635 ;$ g) J. Xiang, P. L. Fuchs, Tetrahedron Lett. 1998, 39, 8597.

[3] a) S. Kim, I. Y. Lee, J.-Y. Yoon, D. H. Oh, J. Am. Chem. Soc. 1996, 118, 5138; b) S. Kim, S. Seguin, J.-Y. Yoon, I. Y. Lee, Synlett 1997, 475; c) S. Kim, J.-Y. Yoon, J. Am. Chem. Soc. 1997 , 119, 5982; d) S. Kim, S. Kim, Bull. Chem. Soc.

[4] a) G. A. Russell, H. Tashtoush, P. Ngoviwatchai, J. Am. Chem. Soc. 1984, 106, 4622; b) G. A. Russell, P. Ngoviwatchai, H. Tashtoush, A. Pla-Dalmau, R. K. Khanna, J. Am. Chem. Soc. 1988, 110,3530 .

[5] a) F. Bertrand, F. Le Guyader, L. Liguori, G. Ouvry, B. Quiclet-Sire, S. Seguin, S. Z. Zard, C. R. Acad. Sci. 2001, 4, 547; b) N. Charrier, S. Z. Zard, Angew. Chem. Int. Ed. 2008, 47, 9443; c) Z. Huang, J. Xu, RSC Adv. 2013, 3, 15114; d) W. Y. Huang, J. Fluor. Chem. 1992, 58, 1.

[6] a) B. Quiclet-Sire, S. Z. Zard, J. Am. Chem. Soc. 1996, 118, 1209; b) F. Le Guyader, B. QuicletSire, S. Seguin, S. Z. Zard, J. Am. Chem. Soc. 1997, 119, 7410; c) B. Quiclet-Sire, S. Z. Zard, Angew. Chem. Int. Ed. 1998, 37, 2864; d) F. Bertrand, B. Quiclet-Sire, S. Z. Zard, Angew. Chem. Int. Ed. 1999, 38, 1943.

[7] a) M. P. Sibi, S. Manyem, Tetrahedron 2000 56, 8033; b) R. Noyori, M. Suzuki, Angew. Chem. Int. Ed. Engl. 1984, 23, 847.

[8] K. Mizuno, M. Ikeda, S. Toda, Y. Otsuji, J. Am. Chem. Soc. 1988, 110, 1288.

[9] a) M. P. Sibi, S. Manyem, J. Zimmerman, Chem. Rev. 2003, 103, 3263; b) M. P. Sibi, N. A. Porter, Acc. Chem. Res. 1999, 32, 163; For Jpn. 2007, 80, 809 . recent applications in total synthesis, see: a) D. Urabe, H. Yamaguchi, M. Inoue, Org. Lett. 2011, 13, 4778; b) D. Urabe, H. Yamaguchi, A. Someya, M. Inoue, Org. Lett. 2012, 14, 3842; c) K. Murai, S.-I. Katoh, D. Urabe, M. Inoue, Chem. Sci. 2013, 4, 2364.

[10] A. P. Schaffner, K. Sarkunam, P. Renaud, Helv. Chim. Acta 2006, 89, 2450

[11] Y. Uenoyama, T. Fukuyama, K. Morimoto, O. Nobuta, H. Nagai, I. Ryu, Helv. Chim. Acta 2006, 89, 2483.

[12] V. Liautard, F. Robert, Y. Landais, Org. Lett. 2011, 13, 2658.

[13] a) S. Kim, C. J. Lim, Angew. Chem., Int. Ed. 2002, 41, 3265; b) Y. Amaoka, M. Nagatomo, M. Watanabe, K. Tao, S. Kamijo, M. Inoue, Chem. Sci. 2014, 5, 4339

[14] a) F. DeVleeschouwer, V. VanSpeybroeck, M. Waroquier, P. Geerlings, F. DeProft, Org. Lett. 2007, 9, 2721; b) N. L. Arthur, P. Potzinger, Organometallics 2002, 21, 2874; c) B. Giese, 'Radical Organic Synthesis: Formation of Carbon-Carbon Bonds', Pergamon Press: Oxford, 1986, Chap. 2, pp 4-35; d) E. Godineau, Y. Landais, Chem. Eur. J. 2009, 15, 3044.

[15] R. Benazzia, V. Liautard, C. Poittevin, B. Ovadia, S. Mohammed, F. Robert, Y. Landais, unpublished results.

[16] a) C. Chatgilialoglu, M. P. Bertrand, C. Ferreri, in 'S-Centered Radicals', Ed. Z. B. Alfassi, Wiley: Chichester, 1999, pp. 312; b) C. Chatgilialoglu, O. Mozziconacci, M. Tamba, K. Bobromski, G. Kciuk, M. P. Bertrand, S. Gastaldi, V. I. Timokhin, J. Phys. Chem. A 2012 , 116, 7623; c) V. L. Timokhin, S. Gastaldi, M. P. Bertrand, C. Chatgilialoglu, J. Org. Chem. 2003, 68, 3532; d) A. Gozdz, P. Maslak, J. Org. Chem. 1991, 56, 2179; e) Y. Takahara, M. Iino, M. Matsuda, Bull. Chem. Soc. Jpn. 1976, 49, 2268.

[17] C. Poittevin, V. Liautard, R. Beniazza, F. Robert, Y. Landais, Org. Lett. 2013, 15, 2814.

[18] a) For a review, see: G. Bernadat, G. Masson, Synlett 2014, 2842; b) F. Drouet, C. Lalli, H. Liu, G. Masson, J. Zhu, Org. Lett. 2011, 13, 94 and references cited therein; c) T. Courant, G. Masson, Chem. Eur. J. 2012, 18, 423; d) J. Jiang, C. Huang, C. Zeng, Y. Zhang, S. Yu, Chem. Eur. J. 2012, 18, 15158 .

[19] X. Ma, D. R. Gang, Nat. Prod. Rep. 2004, 21, 752.

[20] a) M. Rubin, A. Trofimov, V. Gevorgyan, $J$. Am. Chem. Soc. 2005, 127, 10243; b) S. F. Vasilevsky, D. S. Baranov, V. I. Mamatyuk, Y. V. Gatilov, I. V. Alabugin, J. Org. Chem. 2009, 74,6143 .

[21] a) I. Ryu, H. Kuriyama, S. Minakata, M. Komatsu, J.-Y. Yoon, S. Kim, J. Am. Chem. Soc. 1999, 121, 12190; b) S. Kim, K. C. Lim, S. Kim, I. Ryu, Adv. Synth. Catal. 2007, 349, 527.

[22] G. Rouquet, F. Robert, R. Méreau, F. Castet, Y. Landais, Chem. Eur. J. 2011, 17, 13904.

[23] E. Godineau, Y. Landais, J. Am. Chem. Soc. 2007, 129, 12662.

[24] Y. Landais, F. Robert, E. Godineau, L. Huet, N. Likhite, Tetrahedron 2013, 69, 10073.

[25] S. Kim, I. Y. Lee, Tetrahedron Lett. 1998, 39, 1587.

[26] K. Weidner, A. Giroult, P. Panchaud, P. Renaud, J. Am. Chem. Soc. 2010, 132, 17511.

[27] B. Ovadia, F. Robert, Y. Landais, Org. Lett. 2015, 17, 1958.

[28] a) C. Chatgilialoglu, in 'The Chemistry of Sulfones and Sulfoxides', S. Patai, Z. Rappoport, C. J. M. Stirling, Eds.; Wiley: Chichester, 1988, p. 1089; b) M. P. Bertrand, Org. Prep. Proced. Int. 1994, $26,257$.

[29] C. Ollivier, P. Renaud, J. Am. Chem. Soc. 2001, 123,4717

[30] P. Renaud, C. Ollivier, P. Panchaud, Angew. Chem. Int. Ed. 2002, 41, 3460 . 
[31] a) P. Panchaud, C. Ollivier, P. Renaud, S. Zigmantas, J. Org. Chem. 2004, 69, 2755; b) P. Schär, P. Renaud, Org. Lett. 2006, 8, 1569; c) G. Lapointe, K. Schenk, P. Renaud, Org. Lett. 2011, 13, 4774; d) G. Lapointe, K. Schenk, P. Renaud, Chem. Eur. J. 2011, 17, 3207.

[32] S. Cren, P. Schär, P. Renaud, K. Schenk, J. Org. Chem. 2009, 74, 2942.

[33] L. Chabaud, Y. Landais, P. Renaud, Org. Lett. 2002, 4, 4257 .

[34] L. Chabaud, Y. Landais, Tetrahedron Lett. 2003, 44, 6995.

[35] L. Chabaud, Y. Landais, P. Renaud, F. Robert, F. Castet, M. Lucarini, K. Schenk, Chem. Eur. J. 2008, 14, 2744.

[36] L. Chabaud, Y. Landais, P. Renaud, Org. Lett. 2005, 7, 2587.
[37] S. Kim, S. Kim, N. Otsuka, I. Ryu, Angew. Chem. Int. Ed. 2005, 44, 6183.

[38] S. Kim, C. H. Cho, S. Kim, Y. Uenoyama, I. Ryu, Synlett 2005, 3160.

[39] L. Cao, K. Weidner, P. Renaud, Adv. Synth. Catal. 2011, 353, 3467.

[40] a) A. J. Clark, Chem. Soc. Rev. 2002, 31, 1; b) K. Severin, Curr. Org. Chem. 2006, 10, 217.

[41] C. Chatgilialoglu, J. Org. Chem. 1986, 51, 2871.

[42] N. Mantrand, P. Renaud, Tetrahedron, 2008, 64, 11860.

[43] a) B. R. Langlois, E. Laurent, N. Roidot, Tetrahedron Lett. 1991, 32, 7525; b) K. Matcha, A. P. Antonchick, Angew. Chem. Int. Ed. 2014, 53, 11960; c) A. Deb, S. Manna, A. Modak, T. Patra, S. Maity, D. Maiti, Angew. Chem. Int. Ed. 2013, 52, 9747.
[44] a) A. G. O’Brien, A. Maruyama, Y. Inokuma, M. Fujita, P. S. Baran, D. G. Blackmond, Angew. Chem. Int. Ed. 2014, 53, 11868; b) F. O'Hara, D. G. Blackmond, P. S. Baran, J. Am. Chem. Soc. 2013, 135, 12122; c) J. Gui, Q. Zhou, C.-M. Pan, Y. Yabe, A. C. Burns, M. R. Collins, M. A. Ornelas, Y. Ishihara, P. S. Baran, J. Am. Chem. Soc. 2014, 136, 485. 